\title{
Second-Order Statistics of Maximal-Ratio and Equal-Gain Combining in Hoyt Fading
}

\author{
Gustavo Fraidenraich, José Cândido S. Santos Filho, and Michel D. Yacoub
}

\begin{abstract}
Exact expressions for the level crossing rate and average fade duration of $M$-branch equal-gain and maximalratio combining systems in a Hoyt fading environment are presented. The expressions apply to unbalanced, non-identical, independent diversity channels and have been validated by specializing the general results to some particular cases whose solutions are known and, more generally, by means of simulation.
\end{abstract}

Index Terms - Average fade duration, equal-gain combining, Hoyt fading channels, level crossing rate, maximal-ratio combining.

\section{INTRODUCTION}

$\mathbf{T}$ HE performance of wireless communication systems is considerably affected by the multipath propagation phenomena. Diversity-combining techniques are effective means used for mitigating the deleterious effects of fading. The rate of occurrence of fades, or level crossing rate (LCR), and the average fade duration (AFD) provide a dynamic characterization of the communication channel. As second-order statistical quantities, they complement the static probabilistic description of the fading signal (the first-order statistics), and have found several applications in the modelling and design of practical systems.

LCR and AFD expressions of a single channel have been derived for Rayleigh [1], Rice [2], Nakagami- $m$ [3] and, more recently, Hoyt [4] fading environments. Several works have addressed the second-order statistics of diversity-combining systems, including the following. In [5], LCR and AFD expressions of selection combining (SC), maximal-ratio combining (MRC), and equal-gain combining (EGC) for dual-branch diversity in correlated Rayleigh channels were presented. The case of $M$ independent identically distributed (iid) Nakagami$m$ channels was solved in [6] for SC, MRC, and EGC. Some results involving the independent but non-identical Nakagami$m$ diversity case were presented in [7].

The Hoyt (Nakagami-q) distribution [8] spans the range of the fading figure from the one-sided Gaussian to the Rayleigh distributions, and has found applications in mobile satellite propagation channels [4]. Despite its practical interest very little attention has been paid to this type of fading. This paper provides exact LCR and AFD expressions for MRC and EGC in Hoyt channels. The formulas apply to $M$ unbalanced, non-identical, independent branches and have been validated by specializing the general results to some particular cases whose solutions are known and, more generally, by means of simulation.

The authors are with the Department of Communications, State University of Campinas, 13083-970 Campinas, SP, Brazil (e-mail:[michel,gf,candido]@decom.fee.unicamp.br).

\section{BRANCH STATISTICS}

The Hoyt fading envelope $R_{i}$ and phase $\Theta_{i}$ at the $i$ th branch, $i=1, \ldots, M$, is modelled as

$$
\begin{aligned}
R_{i} & =\sqrt{X_{i}^{2}+Y_{i}^{2}} \\
\Theta_{i} & =\arctan \frac{Y_{i}}{X_{i}}
\end{aligned}
$$

where $X_{i}$ and $Y_{i}$ are independent zero-mean Gaussian random variables (RVs) with variances $\sigma_{X_{i}}^{2}$ and $\sigma_{Y_{i}}^{2}$, respectively. The joint probability density function (JPDF) $p_{R_{i}, \Theta_{i}}(\cdot, \cdot)$ of $R_{i}$ and $\Theta_{i}$ is given by [8]

$$
p_{R_{i}, \Theta_{i}}\left(r_{i}, \theta_{i}\right)=\frac{r_{i}}{\Omega_{i} \pi \sqrt{1-b_{i}^{2}}} \exp \left(-\frac{1-b_{i} \cos 2 \theta_{i}}{\Omega_{i}\left(1-b_{i}^{2}\right)} r_{i}^{2}\right)
$$

where $\Omega_{i}=\mathrm{E}\left[R_{i}^{2}\right]$ and $b_{i} \triangleq\left(\sigma_{X_{i}}^{2}-\sigma_{Y_{i}}^{2}\right) /\left(\sigma_{X_{i}}^{2}+\sigma_{Y_{i}}^{2}\right)$, $-1 \leq b_{i} \leq 1$ is the Hoyt fading parameter. The probability density function (PDF) $p_{R_{i}}(\cdot)$ of $R_{i}$ is obtained as

$p_{R_{i}}\left(r_{i}\right)=\frac{2 r_{i}}{\Omega_{i} \sqrt{1-b_{i}^{2}}} \exp \left(-\frac{r_{i}^{2}}{\Omega_{i}\left(1-b_{i}^{2}\right)}\right) I_{0}\left(\frac{b_{i} r_{i}^{2}}{\Omega_{i}\left(1-b_{i}^{2}\right)}\right)$

where $I_{0}(\cdot)$ is the modified Bessel function of the first kind and zeroth order.

In order to derive the LCR and AFD of EGC and MRC, we shall make use of the conditional PDF (CPDF) $p_{\dot{R}_{i} \mid R_{i}, \Theta_{i}}(\cdot \mid \cdot, \cdot)$ of the envelope time derivative $\dot{R}_{i}$ at the $i$ th branch given $R_{i}$ and $\Theta_{i}$. From (1), $R_{i} \dot{R}_{i}=X_{i} \dot{X}_{i}+Y_{i} \dot{Y}_{i}$, where $\dot{X}_{i}$ and $\dot{Y}_{i}$ denote, respectively, the time derivatives of $X_{i}$ and $Y_{i}$. Knowing that $X_{i}=R_{i} \cos \Theta_{i}$ and $Y_{i}=R_{i} \sin \Theta_{i}$, then

$$
\dot{R}_{i}=\dot{X}_{i} \cos \Theta_{i}+\dot{Y}_{i} \sin \Theta_{i}
$$

For isotropic scattering, $\dot{X}_{i}$ and $\dot{Y}_{i}$ are known to be zeromean Gaussian RVs with variances $\dot{\sigma}_{X_{i}}^{2}=\left(\sqrt{2} \pi f_{m}\right)^{2} \sigma_{X_{i}}^{2}$ and $\dot{\sigma}_{Y_{i}}^{2}=\left(\sqrt{2} \pi f_{m}\right)^{2} \sigma_{Y_{i}}^{2}$, respectively, where $f_{m}$ is the maximum Doppler shift in Hertz [1]. Thus, from (5), $\dot{R}_{i}$, given $R_{i}$ and $\Theta_{i}$, is also zero-mean Gaussian distributed, so that

$$
p_{\dot{R}_{i} \mid R_{i}, \Theta_{i}}\left(\dot{r}_{i} \mid r_{i}, \theta_{i}\right)=\frac{1}{\sqrt{2 \pi} \dot{\sigma}_{R_{i}}} \exp \left(-\frac{1}{2}\left(\frac{\dot{r}_{i}}{\dot{\sigma}_{R_{i}}}\right)^{2}\right)
$$

with $\dot{\sigma}_{R_{i}}^{2}=\Omega_{i}\left(\pi f_{m}\right)^{2}\left(1+b_{i} \cos 2 \theta_{i}\right)$.

The LCR and AFD of a random signal are defined, respectively, as the average number of upward (or downward) crossings per second at a given level and as the mean time the signal remains below this level after crossing it in the downward direction. The LCR $n_{R}(r)$ and AFD $T_{R}(r)$ of the 
combiner output $R$ at level $r$ are, respectively, given by [2]

$$
\begin{aligned}
n_{R}(r) & =\int_{0}^{\infty} \dot{r} p_{R, \dot{R}}(r, \dot{r}) d \dot{r} \\
T_{R}(r) & =\frac{P_{R}(r)}{n_{R}(r)}
\end{aligned}
$$

where $p_{R, \dot{R}}(\cdot, \cdot)$ is the JPDF of $R$ and its time derivative $\dot{R}$, and $P_{R}(\cdot)$ is the cumulative distribution function (CDF) of $R$. In the following, (7) and (8) shall be calculated for $M$-branch EGC and MRC in a Hoyt fading environment.

\section{EQUAL-GAIN COMBINING}

In EGC, the received signals are cophased and added so that the combiner output envelope $R$, already taking into account the resultant output noise power, is written as $R=\frac{1}{\sqrt{M}} \sum_{i=1}^{M} R_{i}$. Thus

$$
\dot{R}=\frac{1}{\sqrt{M}} \sum_{i=1}^{M} \dot{R}_{i}
$$

The CDF of $R$ can be calculated as the integration of the JPDF of $R_{i}, i=1, \ldots, M$, over the $M$-dimensional volume bounded by the hyperplane $\sqrt{M} r=\sum_{i=1}^{M} r_{i}$ and the coordinate hyperplanes [9]

$$
\begin{aligned}
P_{R}(r)= & \int_{0}^{\sqrt{M} r} \int_{0}^{\sqrt{M} r-r_{M}} \cdots \int_{0}^{\sqrt{M} r-\sum_{i=3}^{M} r_{i}} \int_{0}^{\sqrt{M} r-\sum_{i=2}^{M} r_{i}} \\
& \times p_{R_{1}, \ldots, R_{M}}\left(r_{1}, \ldots, r_{M}\right) d r_{1} d r_{2} \cdots d r_{M-1} d r_{M} \quad \text { (10) }
\end{aligned}
$$

where $p_{R_{1}, \ldots, R_{M}}\left(r_{1}, \ldots, r_{M}\right)=\prod_{i=1}^{M} p_{R_{i}}\left(r_{i}\right)$ is the JPDF of $R_{i}, i=1, \ldots, M$, since the branches are independent, and $p_{R_{i}}(\cdot)$ is given by (4).

Note, from (6) and (9), that $\dot{R}$, given $R_{i}$ and $\Theta_{i}$, $i=1, \ldots, M$, is a zero-mean Gaussian variate with CPDF

$$
\begin{array}{r}
p_{\dot{R} \mid R_{1}, \ldots, R_{M}, \Theta_{1}, \ldots, \Theta_{M}}\left(\dot{r} \mid r_{1}, \ldots, r_{M}, \theta_{1}, \ldots, \theta_{M}\right)= \\
\frac{1}{\sqrt{2 \pi} \dot{\sigma}_{R}} \exp \left(-\frac{1}{2}\left(\frac{\dot{r}}{\dot{\sigma}_{R}}\right)^{2}\right)
\end{array}
$$

and variance $\dot{\sigma}_{R}^{2}=\frac{\left(\pi f_{m}\right)^{2}}{M} \sum_{i=1}^{M} \Omega_{i}\left(1+b_{i} \cos 2 \theta_{i}\right)$. Next, we shall exploit this fact by including the variates $\Theta_{i} \mathrm{~s}$ in the formulation of $p_{R, \dot{R}}(\cdot, \cdot)$. As shall be seen, this will greatly simplify the calculations. Derivating (10) with respect to $r$ to obtain $p_{R}(r)$ as in [6] and then using the Bayes' rule, $p_{R, \dot{R}}(\cdot, \cdot)$ can be found as (12), where $p_{R_{1}, \ldots, R_{M}, \Theta_{1}, \ldots, \Theta_{M}, \dot{R}}(\cdot, \ldots, \cdot, \cdot, \ldots, \cdot, \cdot)$ is the JPDF of $R_{i}$, $\Theta_{i}, i=1, \ldots, M$, and $\dot{R}$. Of course,

$$
\begin{aligned}
& p_{R_{1}, \ldots, R_{M}, \Theta_{1}, \ldots, \Theta_{M}, \dot{R}}\left(r_{1}, \ldots, r_{M}, \theta_{1}, \ldots, \theta_{M}, \dot{r}\right)= \\
& p_{\dot{R} \mid R_{1}, \ldots, R_{M}, \Theta_{1}, \ldots, \Theta_{M}}\left(\dot{r} \mid r_{1}, \ldots, r_{M}, \theta_{1}, \ldots, \theta_{M}\right) \\
& \quad \times p_{R_{1}, \ldots, R_{M}, \Theta_{1}, \ldots, \Theta_{M}}\left(r_{1}, \ldots, r_{M}, \theta_{1}, \ldots, \theta_{M}\right)
\end{aligned}
$$

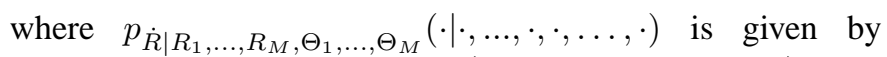
(11) and $p_{R_{1}, \ldots, R_{M}, \Theta_{1}, \ldots, \Theta_{M}}\left(r_{1}, \ldots, r_{M}, \theta_{1}, \ldots, \theta_{M}\right)=$ $\prod_{i=1}^{M} p_{R_{i}, \Theta_{i}}\left(r_{i}, \theta_{i}\right)$, since the branches are independent. Using (13) into (12) and (7), the output LCR of an $M$-branch EGC system in a Hoyt fading environment can be finally written as (14), where $p_{R_{i}, \Theta_{i}}(\cdot, \cdot)$ is given by (3). (Observe that the inclusion of $\Theta_{i} \mathrm{~s}$ in the formulations led to a closed-form integration over $\dot{r}$ in (7).) From (8), (10), and (14), the output AFD of EGC in a multi-branch Hoyt fading is obtained.

\section{Maximal-Ratio Combining}

In MRC, the received signals are cophased, each signal is amplified appropriately for an optimal combining, and the resultant signals are added so that the combiner output envelope $R$ is given by $R=\sqrt{\sum_{i=1}^{M} R_{i}^{2}}$. Thus

$$
\dot{R}=\sum_{i=1}^{M} \frac{R_{i}}{R} \dot{R}_{i}
$$

The MRC analysis follows exactly the same steps detailed for EGC in the previous section, considering that, for MRC, the hyperplane used to compute $P_{R}(\cdot)$ is $r^{2}=\sum_{i=1}^{M} r_{i}^{2}$ and that $\dot{\sigma}_{R}^{2}=\left(\pi f_{m}\right)^{2}\left(\sum_{i=1}^{M} R_{i}^{2}\right)^{-1} \sum_{i=1}^{M} \Omega_{i} R_{i}^{2}\left(1+b_{i} \cos 2 \theta_{i}\right)$. The resulting $P_{R}(\cdot), p_{R, \dot{R}}(\cdot, \cdot)$ and $n_{R}(\cdot)$ are given by (16), (17), and (18), respectively.

$$
\begin{aligned}
P_{R}(r) & =\int_{0}^{r} \int_{0}^{\sqrt{r^{2}-r_{M}^{2}}} \ldots \int_{0}^{\sqrt{r^{2}-\sum_{i=3}^{M} r_{i}^{2}}} \int_{0}^{\sqrt{r^{2}-\sum_{i=2}^{M} r_{i}^{2}}} \\
& \times p_{R_{1}, \ldots, R_{M}}\left(r_{1}, \ldots, r_{M}\right) d r_{1} d r_{2} \ldots d r_{M-1} d r_{M} \quad \text { (16) }
\end{aligned}
$$

As before, $p_{R_{1}, \ldots, R_{M}}\left(r_{1}, \ldots, r_{M}\right)=\prod_{i=1}^{M} p_{R_{i}}\left(r_{i}\right)$, since the branches are independent. From (8), (16), and (18), the output AFD of MRC in multi-branch Hoyt fading is obtained.

\section{RESULTS}

The formulations obtained in this paper can be specialized into those already found in the literature. In particular, for balanced diversity channels and $b_{i}=0, i=1, \ldots, M$, they reduce to the $M$-branch EGC and MRC of the iid Rayleigh case, given by [6, Eqs. 23 and 24] for $m=1$ and [6, Eqs. 38 and 39], respectively. In the same way, for balanced channels and $b_{i} \rightarrow \pm 1, i=1, \ldots, M$, they reduce to the iid one-sided Gaussian case, given by [6, Eqs. 23, 24, 36 and 37] with $m=0.5$. For the more general cases, including identical and non-identical fading branches, exhaustive simulations have been carried out and compared with the analytical expressions obtained here. All the cases investigated revealed an excellent agreement between analytical and simulation results. Figs. 1 and 2 show the LCR and the AFD of EGC and MRC, respectively, for $M=1,2,4$ and $b_{i}=0,0.9,0.999$, considering identical Hoyt-fading channels. For the sake of clarity, the simulation data have been omitted in the figures. In fact, they are practically coincident with the theoretical curves.

\section{CONCLUSIONS}

Exact formulas for level crossing rate and average fade duration of the $M$-branch EGC and MRC techniques in a Hoyt fading environment were presented. These formulas have been validated by specializing the general results to some particular cases whose solutions are known and, more generally, by means of simulation. 


$$
\begin{aligned}
& p_{R, \dot{R}}(r, \dot{r})=\sqrt{M} \int_{0}^{2 \pi} \cdots \int_{0}^{2 \pi} \int_{0}^{\sqrt{M} r} \int_{0}^{\sqrt{M} r-r_{M}} \cdots \int_{0}^{\sqrt{M} r-\sum_{i=3}^{M} r_{i}} \\
& \times p_{R_{1}, R_{2}, \ldots, R_{M}, \Theta_{1}, \ldots, \Theta_{M}, \dot{R}}\left(\left(\sqrt{M} r-\sum_{i=2}^{M} r_{i}\right), r_{2}, \ldots, r_{M}, \theta_{1}, \ldots, \theta_{M}, \dot{r}\right) d r_{2} \cdots d r_{M-1} d r_{M} d \theta_{1} \cdots d \theta_{M} \\
& n_{R}(r)=\sqrt{\pi / 2} f_{m} \int_{0}^{2 \pi} \cdots \int_{0}^{2 \pi} \int_{0}^{\sqrt{M} r} \int_{0}^{\sqrt{M} r-r_{M}} \cdots \int_{0}^{\sqrt{M} r-\sum_{i=3}^{M} r_{i}} \sqrt{\sum_{i=1}^{M} \Omega_{i}\left(1+b_{i} \cos 2 \theta_{i}\right)} \\
& \quad \times p_{R_{1}, \Theta_{1}}\left(\left(\sqrt{M} r-\sum_{i=2}^{M} r_{i}\right), \theta_{1}\right) \prod_{i=2}^{M} p_{R_{i}, \Theta_{i}}\left(r_{i}, \theta_{i}\right) d r_{2} \cdots d r_{M-1} d r_{M} d \theta 1 \cdots d \theta_{M}
\end{aligned}
$$

$$
\begin{aligned}
& p_{R, \dot{R}}(r, \dot{r})=\int_{0}^{2 \pi} \ldots \int_{0}^{2 \pi} \int_{0}^{r} \int_{0}^{\sqrt{r^{2}-r_{M}^{2}}} \ldots \int_{0}^{\sqrt{r^{2}-\sum_{i=3}^{M} r_{i}^{2}}} \\
& \times \frac{r}{\sqrt{r^{2}-\sum_{i=2}^{M} r_{i}^{2}}} p_{R_{1}, R_{2}, \ldots, R_{M}, \Theta_{1}, \ldots, \Theta_{M}, \dot{R}}\left(\left(\sqrt{r^{2}-\sum_{i=2}^{M} r_{i}^{2}}\right), r_{2}, \ldots, r_{M}, \theta_{1}, \ldots, \theta_{M}, \dot{r}\right) d r_{2} \cdots d r_{M-1} d r_{M} d \theta 1 \cdots d \theta_{M}
\end{aligned}
$$

$$
\begin{array}{r}
n_{R}(r)=\sqrt{\pi / 2} f_{m} \int_{0}^{2 \pi} \ldots \int_{0}^{2 \pi} \int_{0}^{r} \int_{0}^{\sqrt{r^{2}-r_{M}^{2}}} \ldots \int_{0}^{\sqrt{r^{2}-\sum_{i=3}^{M} r_{i}^{2}}} \sqrt{\frac{\Omega_{1}\left(r^{2}-\sum_{i=2}^{M} r_{i}^{2}\right)\left(1+b_{1} \cos 2 \theta_{1}\right)+\sum_{i=2}^{M} \Omega_{i} r_{i}^{2}\left(1+b_{i} \cos 2 \theta_{i}\right)}{r^{2}-\sum_{i=2}^{M} r_{i}^{2}}} \\
\left.\quad \times p_{R_{1}, \Theta_{1}}\left(\sqrt{r^{2}-\sum_{i=2}^{M} r_{i}^{2}}\right), \theta_{1}\right) \prod_{i=2}^{M} p_{R_{i}, \Theta_{i}}\left(r_{i}, \theta_{i}\right) d r_{2} \cdots d r_{M-1} d r_{M} d \theta 1 \cdots d \theta_{M} \quad(18)
\end{array}
$$

\section{REFERENCES}

[1] W. C. Jakes. Microwave Mobile Communications. New York: Wiley, 1974.

[2] S. O. Rice. Mathematical analysis of random noise. Bell System Technical Journal, 23:282-332, Jul. 1944.

[3] M.D. Yacoub, J. E. Vargas B. and Leonardo G. R. Guedes. On High Order Statistics of the Nakagami-m Distribution. IEEE Trans. Veh. Technol., 48(3):790-793, May 1999.

[4] Cheng-Xiang Wang, Neji Youssef and Matthias Patzold. Level-crossing rate and average duration of fades of deterministic simulation models for Nakagami-Hoyt fading channels. In The 5th International Symposium on Wireless Personal Multimedia Communications, volume 1, pages 272276, Oct. 2002.

[5] F. Adachi, M. T. Feeney and J. D. Parsons. Effects of correlated fading on level crossing rates and average fade durations with predectection diversity reception. In Proc. Inst. Elect. Eng., pages 11-17, Feb. 1998.

[6] M.D. Yacoub, C.R.C.M. da Silva and J. E. Vargas B. Second-order statistics for Diversity-Combining Techniques in Nakagami-Fading Channels. IEEE Trans. Veh. Technol., 50(6):1464-1470, Nov. 2001.

[7] Cyril-Daniel Iskander and P. Takis Mathiopoulos. Analytical Level Crossing Rates and Average Fade Durations for Diversity Techniques in Nakagami Fading Channels. IEEE Trans. Commun., 50(8):1301-1309, Aug. 2002.

[8] R. S. Hoyt. Probability Functions for the Modulus and Angle of the Normal Complex Variate. Bell System Technical Journal, 26:318-359, Apr. 1947.

[9] D. G. Brennan. Linear Diversity Combining Techniques. In IRE, volume 47, pages 1075-1102, Jun. 1959.

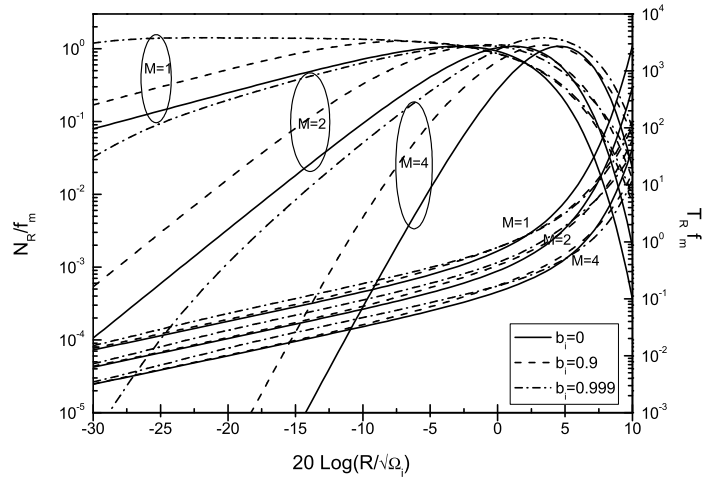

Fig. 1. LCR and AFD of EGC for identical Hoyt-fading channels $\left(M=1,2,4\right.$ and $\left.b_{i}=0,0.9,0.999\right)$.

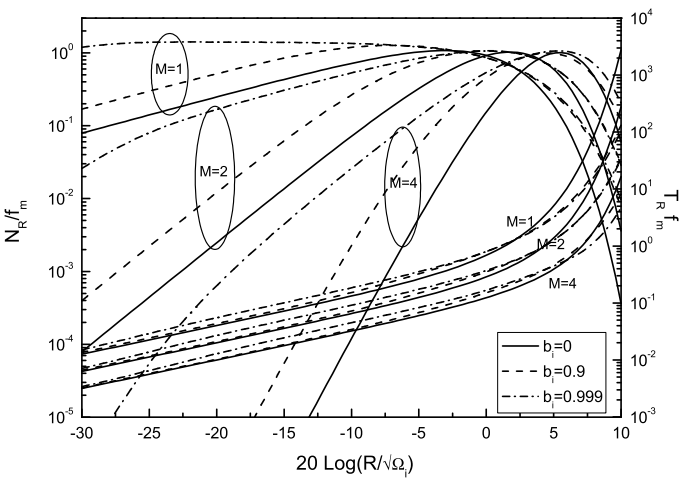

Fig. 2. LCR and AFD of MRC for identical Hoyt-fading channels ( $M=1,2,4$ and $\left.b_{i}=0,0.9,0.999\right)$. 